\title{
Trap identification of the constitutive promoter-like sequences from the bacterial fish pathogen, as exemplified by Edwardsiella tarda
}

\author{
Sang Yoon Lee, Ki Hong Kim*, Dong Soo Kim and Yoon Kwon Nam ${ }^{\dagger}$ \\ Department of Marine Bio-Materials \& Aquaculture, Pukyong National University, Busan 608-737, Korea \\ *Department of Aquatic Life Medicine, Pukyong National University, Busan 608-737, Korea
}

\begin{abstract}
A trap identification system for isolating functional sequences to allow the constitutive expression of foreign protein from Edwardsiella tarda was developed. Using the green fluorescent protein (GFP) reporter-based trap system, various functional sequences to drive heterologous expression of the GFP were selectable in Escherichia coli host. However from the bioinformatic sequence analysis, all the segments predicted as regulatory regions were not native promoters actually existing upstream of endogenous E. tarda genes. Instead, a number of non-authentic sequences, possibly resulted from the random shuffling and/or intermolecular ligation were also proven to be able to display a potent GFP expression in the recombinant E. coli. Further analysis with selected clones showed that both authentic and non-authentic sequences could function in as a constitutive promoter, leading quite a consistent and stable GFP expression after repetitive subcultures. Microscopic examination also confirmed the uniform pattern of GFP expression in every host bacterium. Semi-quantitative assay of GFP showed that there was no clear relationship between expression levels and organizational features of the promoters trapped. Functional promoter-like elements achieved in the present study could be a good starting material for multivalent genetic engineering of $E$. tarda in order to produce recombinant vaccines in a cost-effective fashion.
\end{abstract}

Key words: Constitutive expression, Edwardsiella tarda, Promoter trap

Vaccination-based prevention against a specific disease has already been indispensable with the health control of many farmed fish species (Lorenzen, 1999; Adams and Thompson, 2006). Compared to the vaccination of terrestrial animals, administration of the vaccines to aquaculture-relevant species should often include a greater number of individuals. Hence, the efficient expression system allowing the cost-effective production of the recombinant vaccines is one of pivotal prerequisites for successful adoption of the vaccines in the farming

†Corresponding author : Yoon Kwon Nam

Tel : +82-51-629-5918

E-mail : yoonknam@pknu.ac.kr practice of the aquacultured fishes (Secombes, 2008).

Edwardsiella septicaemia, caused by infection with a Gram negative bacterium Edwardsiella tarda, has been one of the major causes of mass mortalities in cultured fish (Thune et al., 1993; Plumb, 1999). Recently, recombinantly inactivated vaccine types of $E$. tarda, such as ghost and cadaver bacteria, have been reported as an alternative to classical heat-or formalin-inactivated vaccines (Kwon et al., 2005, 2006, 2007 and 2009; Lee et al., 2008). In addition, the utilization of the ghost or cadaver E. tarda as a delivery tool for heterologous antigens was reported recently (Choi et al., 2010). 
Most post-mortem approaches for fish vaccination have been based on the use of tightly inducible promoters, in which the transcription of the antigen-coding gene was activated by the addition of a chemical inducer (e.g., isopropyl B-D-1-thiogalactopyranoside; for lac or tac promoters) (Plant et al., 2009; Qin et al., 2010) or the thermal elevation of bacterial culture (e.g., for lambda $\mathrm{P}_{\mathrm{R}} / \mathrm{CI}$ regulatory system) (Kwon et al., 2006; Ningqiu et al., 2008). Although these expression systems have been widely successful in laboratory applications, the use of such systems in a commercial scale may often cause the increase of the production cost for the vaccines due to their needs of a priced inducer compound or a specific apparatus for the programmed thermal control of the bacterial culture.

For this reason, the development of alternative expression system with novel regulatory element(s), which is able to drive the expression of recombinant proteins during the normal growth phase of the host cells (i.e., a constitutive expression), might also be required in certain circumstances, although the constitutively expressed recombinant proteins should have no adverse effect on the viability and growth of the hosts (Gat et al., 2003; Brondyk, 2009). However, the functional sequences to drive constitutive expression have been little exploited for developing the recombinant fish vaccines. In line with our long-term goal to generate efficient expression systems to produce fish vaccines in a cost-effective fashion, the objective of this study was to generate the trap identification system for isolating and characterizing the functional sequences capable of driving the constitutive expression of foreign protein from a known bacterial fish pathogen, Edwardsiella tarda.

\section{Materials and Methods}

Bacterial species, culture conditions, nucleic acid preparation and enzymes

A donor bacterial species, Edwardsiella tarda (Gram-) used in this study was the FSW910410 strain (Kwon et al., 2005), while an Escherichia coli strain (XL1-blue MRF'; Stratagene, La Jolla, CA, USA) was used as a recipient platform for the trap identification of promoter-like sequences. Bacterial cells were grown in Luria Broth (LB; Difco Laboratories, Detroit, USA) at 28 (for E. tarda) to $37{ }^{\circ} \mathrm{C}$ (for E. coli). Transformed E. coli cells were grown in LB supplemented with 100 $\mu \mathrm{g} / \mathrm{ml}$ of ampicillin (Sigma-Aldrich, St. Louis, MO, USA). Genomic DNA from the E. tarda was prepared using the LaboPass ${ }^{\mathrm{TM}}$ Mini Kit (Cosmo Genetech, Seoul, Korea). Purification of plasmid DNA from recombinant E. coli was carried out using the AccuPrep ${ }^{\circledR}$ Plasmid Extraction Kit (Bioneer, Daejeon, Korea). All the procedures for nucleic acid preparations were conducted according to the manufacturers' instructions. Polymerase chain reactions in this study were carried out using the Expand High Fidelity PCR System (Roche Applied Science, Mannheim, Germany), and restriction endonucleases used in this study were purchased from New England Biolabs (NEB; Ipswich, MA, USA).

\section{Construction of promoter trap vector}

The trap vector was constructed with the pUC18 (Stratagene) as a backbone plasmid (Fig. 1). From the pUC18 backbone, lac promoter and lacZ' fragment including multiple-cloning site (MCS) were eliminated by site-directed PCR of the selective region using a 
pair of PCR primers (puc18F and puc18R). Each primer contained the restriction sequence (CCCGGGTACC for puc18F and CCCGGG for puc18R; recognized by the restriction enzymes $X m a \mathrm{I}-K p n \mathrm{I}$ and $X m a \mathrm{I}$, respectively). Information on oligonucleotide primers and thermal cycling conditions used in this study was provided in Table 1. Amplified product was digested by XmaI and self-ligation was induced in order to generate the backbone plasmid, pUC18' lacking the unnecessary lacZ' cassette. The pUC18' was digested with KpnI and treated with calf alkaline phosphatase (Roche Applied Science) according to the manufacturer's instruction. To prepare the insert reporter gene, GFPuv gene was isolated from a commercially available plasmid (pGFPuv; Clontech Laboratories Inc., Mountain View, CA, USA) by PCR. For the PCR amplification, forward (GFPuvF) and reverse (GFPuvR) primers were designed to contain $K p n I-B g l I I-X b a \mathrm{I}$ and EcoRI-KpnI sites at 5'-end of each primer, respectively. Additionally the forward primer GFPuvF was designed to possess a ribosomal binding site (RBS; 5'-AAGGAGATATACAT-3') (Valdivia and Falkow, 1997) to facilitate the translation of the GFPuv gene transcripts. The amplified product (GFPuv fragment) was TA cloned using the pGEM®-T Easy Vector System (Promega, Madison, WI, USA) and spliced again from the T-vector by the digestion with KpnI. Ligation between the KpnI-digested insert (GFPuv) and KpnI-linearized pUC18' plasmid was performed at 12 ${ }^{\circ} \mathrm{C}$ for $12 \mathrm{~h}$ in a reaction volume of $10 \mu \mathrm{l}$ using the
T4 DNA ligase (Promega). The conformation of the resultant promoter-less GFPuv vector $(3.1 \mathrm{~kb}$; named as pGFPuv) was confirmed by sequencing analysis. The trap vector possessed four restriction sites (XmaI-KpnI$B g l I I-X b a \mathrm{I})$ just before the RBS introduced upstream of the GFPuv structure gene (Fig. 1). The pGFPuv' was transformed to E. coli XLI-Blue MRF' and the absence of green fluorescence in the transformed bacteria was confirmed under UV light (photograph not shown).

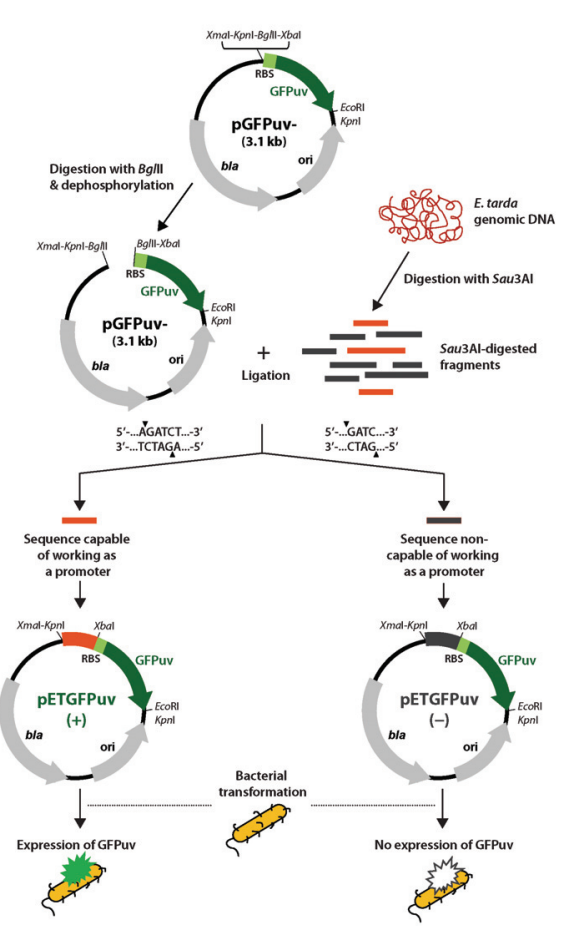

Fig. 1. Construction of promoter-less GFPuv trap vector and trap strategy of promoter-like sequences from Edwardsiella tarda. Sequences of oligonucleotide primers (puc18F/R noted on $\mathrm{pUC18}$ and GFPuvF/R on pGFPuv) are provided in Table 1. 
Table 1. Oligonucleotide primers and thermal cycling conditions used in this study.

\begin{tabular}{llll}
\hline Name & Sequence $\left(5^{\prime} \text { to } 3^{\prime}\right)^{1}$ & Purpose & PCR cycling condition ${ }^{2}$ \\
\hline
\end{tabular}

$\begin{array}{lll}\text { puc18F } & \text { ATTCCCGGGTACCAGAGGCGGTTTGCGTATT } & \text { Generation of plasmid } \\ \text { puc18R } & \text { ATATCCCGGGTGATGCGGTATTTTCTCCTTACGCATC } & \begin{array}{l}30 \text { cycles at } 94{ }^{\circ} \mathrm{C} \text { for } \\ 30 \mathrm{~s}, 58{ }^{\circ} \mathrm{C} \text { for } 45 \mathrm{~s} \text { and }\end{array} \\ 72{ }^{\circ} \mathrm{C} \text { for } 1 \mathrm{~min}\end{array}$

GFPuvF GGTACCAGATCTAGAAGGAGATATACATATGAGTA Isolation of GFPuv 30 cycles at $94{ }^{\circ} \mathrm{C}$ for AAGGAGAAGAACT gene and addition of $30 \mathrm{~s}, 56{ }^{\circ} \mathrm{C}$ for $30 \mathrm{~s}$ and

GFPuvR GGTACCGAATTCATTATTTGTAGAGCTCATCCATGCC ribosomal binding site $72{ }^{\circ} \mathrm{C}$ for 1 min

pucSEQF GAGACGGTCACAGCTTGTCT Sequencing of insert

pucSEQR TTGCATCACCTTCACCCTCT DNA trapped

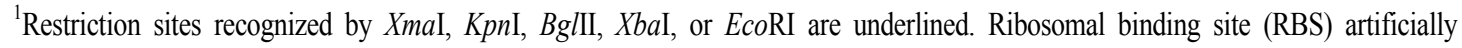
introduced to upstream of GFPuv gene is noted by boldface letters.

${ }^{2}$ Each PCR reaction was accompanied by initial denaturation step at $94{ }^{\circ} \mathrm{C}$ for $3 \mathrm{~min}$ and final elongation step at $72{ }^{\circ} \mathrm{C}$ for $5 \mathrm{~min}$.
}

Construction of trap library with $E$. tarda genomic DNA

Five $\mu \mathrm{g}$ of purified E. tarda genomic DNA was digested with 3 units of Sau3AI at $37^{\circ} \mathrm{C}$ for $2 \mathrm{~h}$. The digest was run on $1 \%$ agarose gel and DNA fragments with lengths of approximately 200 to $500 \mathrm{bp}$ were recovered with QIAEX II Gel Extraction Kit (Qiagen, Hilden, Germany). On the other hand, the trap vector pGFPuv was digested with $B g l I I$ to generate the compatible ends with the genomic fragments digested with Sau3AI. The linearized plasmid was treated with calf alkaline phosphatase (Roche Applied Science) for the dephosphorylation of the ends, and then gel purified using QIAEX II Gel Extraction Kit (Qiagen). The Sau3AI-digested E. tarda genomic fragments were ligated into the trap vector (average molar ratio of vector : insert $=1: 3)$ as described above. When the ligation reaction completed, E. coli XL1-blue MRF' strain was transformed with the reaction mixture using a conventional $\mathrm{Ca}^{2+}$ transformation method. For the selective growth of recombinant bacteria, the bacterial mixture was spread onto a LB agar (1.5\%) plate supplemented with $100 \mu \mathrm{g} / \mathrm{ml}$ of ampicillin (Sigma-Aldrich). After overnight culture, the bacterial colonies were subjected to the fluorescent image analysis using the Quantity-One ${ }^{\mathrm{TM}}$ image analysis software implemented in VersaDoc 4000 (Bio-Rad, Hercules, CA, USA). Three replicated traps were performed, and the GFPuv-expressing bacterial clones were selected for further validations.

Sequence analysis and identification of promoter region Insert DNA trapped in pGFPuv was sequenced at both directions with two sequencing primers (pucSEQF and 
pucSEQR; Table 1), which were complementary to either ends of trap insertion site in the pGFPuv. Raw sequence data were edited by Sequencher (ver. 4.2; Gene Codes, Ann Arbor, MI, USA). Bioinformatic prediction of promoter-like sequence was carried out using the web tools, SOFTBERRY (http://linux1.softberry.com/berry.phtml) and FRUITFLY (http://www.fruitfly.org/seq tools/promoter .html). Trapped sequences were also subjected to BLAST search against the E. tarda genome sequence (NC_013508; http://ncbi.nlm.nih.gov/genome) for identifying the location of the trapped segments in the E. tarda genome.

\section{Validation of constitutive GFP expression}

Based on the probability scores from bioinformatic predictions as well as preliminary fluorescence image analysis, representative clones harboring authentic or non-canonical promoters were selected. To validate their capabilities to drive constitutive expressions, each clone was grown up to 1.0 of optical density (at $\mathrm{OD}_{600 \mathrm{~nm}}$ ) at $37^{\circ} \mathrm{C}$. An aliquot ( $1.6 \times 10^{9}$ cells $)$ of each bacterial culture were transferred to a 96 -well plate for measuring the fluorescent intensity using the XFLUOR4 fluorescence analysis software (ver. 4.2) implemented in Polarion fluorometer (Tecan Group Ltd., Männedorf, Switzerland). As a negative control, the bacterial clone harboring the promoter-less pGFPuv plasmids was also prepared identically in order to subtract the background fluorescence signal. Triplicate assays were performed in an independent manner. Uniform expression of GFPuv in the recombinant bacteria was checked by microscopic examination. Bacterial cells suspended in LB was placed onto a clean slide glass and covered with a UV-transmittable cover slip. GFPuv-expressing bacterial cells were visualized and analyzed by the MetaVue ${ }^{\mathrm{TM}}$ Imaging System (ver. 6.1; Molecular Devices, Downingtown, PA, USA) equipped in the ECLIPSE E400 fluorescence microscope (Nikon Corporation Instruments, Japan). Finally to examine any effects of the overexpressed GFPuv on the growth of XL1-blue MRF', the growth curves of selected recombinant bacterial clones $(n=10)$ and negative control clones carrying pGFPuv' $(n=5)$ were compared each other. Freshly grown bacterial colony was inoculated into $10 \mathrm{ml}$ of LB supplemented with ampicillin $(100 \mu \mathrm{g} / \mathrm{ml})$ and cultured at $37{ }^{\circ} \mathrm{C}$ with a constant shaking at $300 \mathrm{rpm}$. After 5, 10, 15 and 20 $\mathrm{h}$ of the inoculation, the bacterial growth was measured based on the optical density at $600 \mathrm{~nm}\left(\mathrm{OD}_{600 \mathrm{~nm}}\right)$.

\section{Statistics}

Differences in the levels of GFPuv expression among the clones were assessed by ANOVA followed by Duncan's multiple range tests, while the difference in the growth rates between GFPuv-expressing recombinants and naïve $E$. coli were tested with Student's $t$-test by using SPSS software (ver. 10.1.3). Difference was considered to be significant when $p<0.05$.

\section{Results and Discussion}

Out of approximately $5 \times 10^{4}$ colonies, over one hundred clones showing clear GFP expression were identified. Of those clones, 60 relatively well-separated clones were randomly picked up and streaked again onto each of new LB plate for isolating single colony of GFPuv-expressing recombinant bacteria. After two rounds of successive isolation, they represented quite variable 
levels of GFPuv expression one another based on the fluorescence image analysis with VersaDoc (Bio-Rad), suggesting that various promoter-like sequences with differential potencies were trapped from the E. tarda genome. Owing to their strong GFP expression, many of bacterial cultures from those recombinant clones could be distinguishable from naïve $E$. coli and negative control clone carrying only pGFPuv with naked eye even under daylight condition (photograph not shown).

From the bioinformatic sequence analysis with the 60 selected clones, most, but not all, sequences were predicted to possess promoter-like element(s) with differential probability scores ranging from 0.80 to 1.00 . However, all the segments predicted as regulatory regions were not native promoters actually existing upstream of endogenous $E$. tarda genes (Fig. 2). When analyzed by BLAST search against the $E$. tarda genome database, only a few clones (less than 20\%) were proven to possess segments containing the authentic sequences recognized as native promoters of known or hypothetical genes. In contrast, remaining a significant portion of clones revealed the non-authentic sequences. In overall, the clones examined in this study were broadly categorized into five groups based on the organizational characteristics of the trapped segments: (I) isolated potentially authentic promoters located at the upstream of open reading frame (ORF) either for annotated or hypothetical gene (for example, the clone G02 in Fig. 2), (II) trapped as a fused form between native promoter region and the considerable length of following ORF fragment (e.g., G21), (III) trapped an internal ORF segment to resemble promoter sequences (e.g., G11), (IV) formed promoter-like sequences by fusion of more than two ORF fragments from different genes (e.g., G27), and $(\mathrm{V})$ trapped 3'-untranslated region (UTR) of a certain gene to resemble promoter (e.g., G03). As shown in Fig. 2, many of these non-authentic sequences would be resulted from intermolecular fusion(s) of the Sau3AI-digested inserts prior being incorporated into the trap vector during ligation step. It undoubtedly suggests that trapped promoters isolated from the non-sequenced prokaryotic genome should be carefully

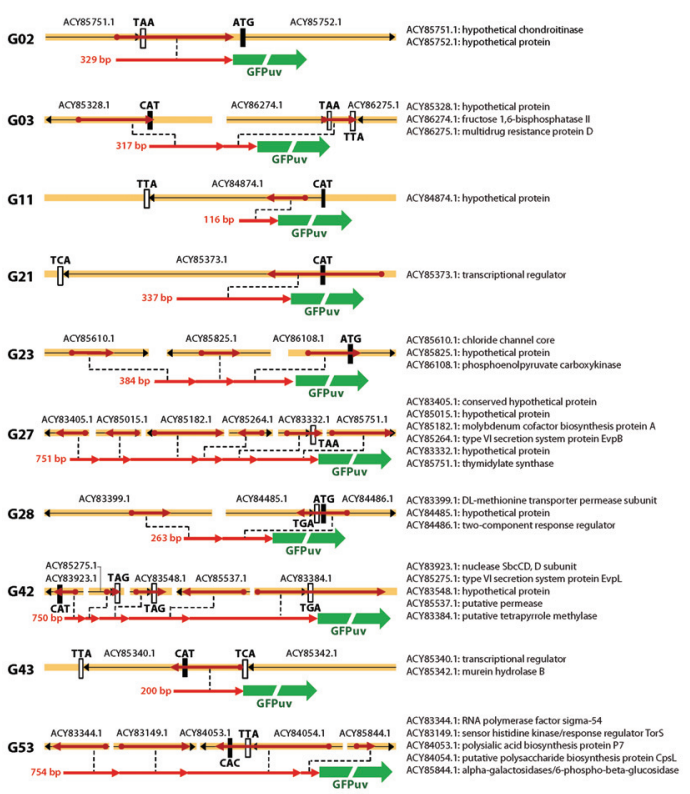

Fig. 2. Chromosomal arrangement and location of the trapped segments in the Edwardsiella tarda genome. Each trapped sequence was subjected to BLAST search against the $E$. tarda genome (NC_013508), and representative 10 clones selected are shown. Sites for start codons predicted in NC_013508 are indicated by closed vertical boxes while the stop codons by open vertical boxes. The orientation (transcriptional direction) of each gene in the genome is indicated by the thin horizontal arrow, while trapped fragments are noted by the thick arrows. Diagrams are not drawn to scale the GFPuv gene in the trap vector. Annotations of genes are also shown in right with accession numbers. 
validated if they would be authentic and native promoters (Dunn and Handelsman, 1999; Doree and Mulks, 2001; Rasko et al., 2007; Horzempa et al., 2008) since promoter-like activity could be readily achieved in the heterologous expression with non-canonical sequences only to resemble the recognizable promoters.

Despite their unusual organizations, the expressions were quite consistent and stable after a number of repetitive subcultures (at least ten times) (Fig. 3A). Microscopic examination also confirmed the uniform pattern of GFPuv expression in every bacterium (Fig. 3B). Based on the semi-quantitative assay of GFPuv expression, there was no clear relationship between expression levels and organizational features of the promoters trapped. Furthermore, a number of such non-authentic sequences drove the expression which was highly comparable to those observed with endogenous authentic promoters (Fig. 3C). From the comparative examination of growth rates between GFPuv-expressing bacteria and negative control bacterial carrying pGFPuv, no notable difference was observed regardless the five detection points up to $20 \mathrm{~h}$ after inoculation, although further evaluation to test effects of various foreign proteins including actual antigenic proteins on the viability and growth rate of the bacterial hosts should be needed (Fig. 3D). Currently, detailed mechanism behind this finding has not been yet understood; hence further finer typing would be needed to better understand how those sequences are able to drive such a strong and constitutive expression of the downstream foreign gene. Extensive tailoring and functional validation with the differentially selected region(s) from each clone would be valuable to get a deeper insight into the mechanism of this issue. Nevertheless, our data may suggestive of the possibility that artificial random shuffling between genomic segments could be a potentially useful mean to generate functional sequences to resemble the authentic promoters, which are capable of driving a powerful prokaryotic expression in a constitutive fashion. We also made similar findings (i.e., trap of the non-authentic but functional promoter-like segments) with other bacterial donor species such as Vibrio anguillarum (Gram-) and Streptococcus iniae $(\mathrm{Gram}+)$ based on the present promoter trap strategy (unpublished data). The genomic elements trapped in this study represent potential utility as constitutive promoters for the efficient production of foreign proteins with various research purposes. Furthermore when considering that $E$. tarda is one of the most important target pathogens against which protective vaccines should be developed (Sun et al., 2009), the functional promoter-like elements achieved in the present study could also be good starting materials for multivalent genetic engineering of $E$. tarda in order to produce recombinant vaccines in a cost-effective fashion (Lee et al., 2008; Choi et al., 2010). Further study should be followed in order to address a number of biotic and abiotic factors affecting their conditional regulations for the heterologous expression of target antigenic proteins (Rollenhagen and Bumann, 2006). 
A)

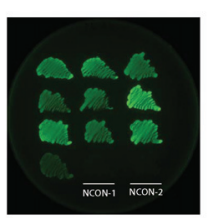

B)

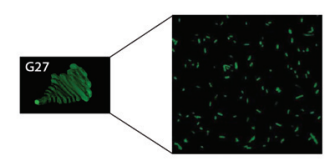

C)

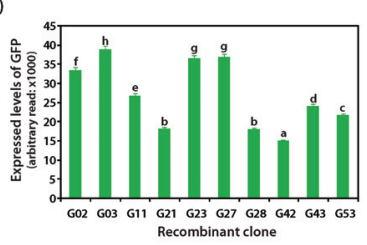

D)

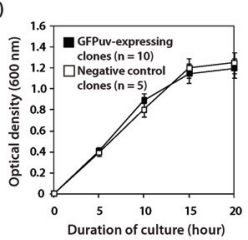

Fig. 3. Expression and growth of recombinant Escherichia coli harboring the GFPuv expression vector driven by Edwardsiella tarda promoter-like sequences. (A) Bacterial smear of randomly chosen GFPuv-expressing clones on LB agar plate along with two negative control clones (NCON-1 and-2) harboring the promoter-less pGFPuv plasmid (B) A representative microscopic image of bacterial cells obtained from the clone G27 in Fig. 2 in order to show the uniform expression of GFPuv (C) Fluorometric analysis of GFPuv expression levels among selected clones. The expression level of each GFPuv-expressing clone was subtracted by the background level detected in the negative control clone carrying pGFPuv-. Means based on triplicate examinations are noted by histograms and standard deviations by $\mathrm{T}$ bars. Means with different letters are significantly different based on ANOVA followed by Duncan's multiple range test ( $p$ $<0.05$ ). (D) Growth curves of GFPuv-expressing clones ( $n$ $=10)$ and negative controls carrying pGFPuv ${ }^{-}(n=5)$. At each detection point, no significant difference was observed based on Student's $t$-test $(p<0.05)$.

\section{Acknowledgement}

This study was supported by a research fund from the Ministry of Land, Transport and Maritime Affairs, Republic of Korea (Project \#20088033-1).

\section{References}

Adams, A. and Thompson, K.D.: Biotechnology offers

revolution to fish health management, Trends Biotechnol., 24:201-205, 2006.

Brondyk, W.H.: Selecting an appropriate method for expressing a recombinant protein, Meth. Enzymol., 463:131-147, 2009.

Choi, S.H., Nam, Y.K. and Kim, K.H.: Novel expression system for combined vaccine production in Edwardsiella tarda ghost and cadaver cells, Mol. Biotechnol., 46:127-133, 2010.

Doree, S.M. and Mulks, M.H.: Identification of an Actinobacillus pleuropneumoniae consensus promoter structure, $\mathrm{J}$. Bacteriol., 183:1983-1989, 2001.

Dunn, A.K. and Handelsman, J.: A vector for promoter trapping in Bacillus cereus, Gene, 226: 297-305, 1999. Gat, O., Inbar, I., Aloni-Grinstein, R., Zahavy, E., Kronman, C., Mendelson, I., Cohen, S., Velan, B. and Shafferman, A: Use of a promoter trap system in Bacillus anthracis and Bacillus subtilis for the development of recombinant protective antigen-based vaccines, Infect. Immun., 71:801-813, 2003.

Horzempa, J., Tarwacki, D.M., Carlson, P.E., Robinson, C.M. and Nau, G.J.: Characterization and application of a glucose-repressible promoter in Francisella tularensis, Appl. Environ. Microbiol., 74:2161-2170, 2008.

Kwon, S.R., Kang, Y.J., Lee, D.J., Nam, Y.K., Kim, S.K. and K.H. Kim.: Generation of Vibrio anguillarum ghost by coexpression of PhiX 174 lysis E gene and staphylococcal nuclease A gene, Mol. Biotechnol. 42:154-159, 2009.

Kwon, S.R., Lee, E.H., Nam, Y.K., Kim, S.K. and Kim, K.H.: Efficacy of oral immunization with Edwardsiella tarda ghosts against edwardsiellosis 
in olive flounder (Paralichthys olivaceus), Aquaculture, 269:84-88, 2007.

Kwon, S.R., Nam, Y.K., Kim, S.K. and Kim, K.H.: Protection of tilapia (Oreochromis mosambicus) from edwardsiellosis by vaccination with Edwardsiella tarda ghosts, Fish Shellfish Immunol., 20:621-626, 2006.

Kwon, S.R., Nam, Y.K., Kim, S.K., Kim, D.S. and Kim, K.H.: Generation of Edwardsiella tarda ghosts by bacteriophage, Aquaculture, 250:16-21, 2005.

Lee, D.J., Kwon, S.R., Kosuke, Z., Lee, E.H., Nam, Y.K., Kim, S.K. and K.H. Kim.: Generation of safety enhanced Edwardsiella tarda ghost vaccine, Dis. Aquat. Org., 81:249-254, 2008.

Lorenzen, N.: Recombinant vaccines: experimental and applied aspects, Fish Shellfish Immunol., 9:361-365, 1999.

Ningqiu, L., Junjie, B., Xiaozhe, F., Haihua, L., Xing, Y. and Cunbin, S.: An outer membrane protein, OmpK, is an effective vaccine candidate for Vibrio harveyi in orange-spotted grouper (Epinephelus coioides), Fish Shellfish Immunol., 25:829-833, 2008.

Plant, K.P., LaPatra, S.E. and Cain, K.D.: Vaccination of rainbow trout, Oncorhynchus mykiss (Walbaum), with recombinant and DNA vaccines produced to Flavobacterium psychrophilum heat shock proteins 60 and 70, J. Fish Dis., 32:521-534, 2009.

Plumb, J.A.: Edwardsiella septicaemias, Viral, bacterial and fungal infections. In: Fish Diseases and Disorders, pp. 479-521, Woo, P.T.K. and Bruno
E.W., CABI, Wallingford, 1999.

Qin, H., Jin, X., Huang, W. and Liu, Y.: Production of an anti-idiotypic antibody single chain variable fragment vaccine against Edwardsiella tarda, Acta Biochim. Biophys. Sin., 42: 129-136, 2010. Rasko, D.A., Esteban, C.D. and Sperandio, V.: Development of novel plasmid vectors and a promoter trap system in Francisella tularensis compatible with the pFLN10 based plasmids, Plasmid, 58:159-166, 2007.

Rollenhagen, C. and Bumann, D.: Salmonella enterica highly expressed genes are disease specific, Infect. Immun., 74:1649-1660, 2006.

Secombes, C.: Will advances in fish immunology change vaccination strategies, Fish Shellfish Immunol., 25:409-416, 2008.

Sun, K., Wang, H., Zhang, M., Xiao, Z.Z. and Sun, L.: Genetic mechanisms of multi-antimicrobial resistance in a pathogenic Edwardsiella tarda strain, Aquaculture, 289:134-139, 2009.

Thune, R.L., Stanley, L.A. and Cooper, R.K.: Pathogenesis of gram negative bacterial infections in warm water fish, Ann. Rev. Fish Dis., 3:37-68, 1993.

Valdivia, R.H. and Falkow, S.: Fluorescence-based isolation of bacterial genes expressed within host cells, Science, 277:2007-2011, 1997.

\footnotetext{
Manuscript Received : September 7, 2011

Revised : September 19, 2011 Accepted : September 19, 2011
} 